SCIENTIFIC LETTER

\title{
Decrease in circulating endothelial progenitor cells in patients with stable coronary artery disease
}

\author{
T Eizawa, U Ikeda, Y Murakami, K Matsui, T Yoshioka, M Takahashi, K Muroi, K Shimada
}

Heart 2004;90:685-686. doi: 10.1136/hrt.2002.008144

T he bone marrow derived endothelial progenitor cells (EPCs) are considered to originate from haematopoietic stem cells, which are positive for CD34. ${ }^{1}$ Human CD34 expressing cells $\left(\mathrm{CD} 34^{+}\right.$cells) injected into nude mice and rats undergoing neovascularisation caused by hindlimb ischaemia are incorporated into the neovasculature and express endothelial antigens. We recently reported that transplantation of autologous bone marrow cells, including $\mathrm{CD}^{+} 4^{+}$cells, improved ischaemia in patients with critical limb ischaemia. ${ }^{2}$ Moreover, increased neovascularisation by bone marrow derived $\mathrm{CD}_{3} 4^{+}$cells was shown to improve cardiac function.

Recently, a mobilisation of EPCs into circulation from bone marrow was reported in patients with acute myocardial infarction and acute coronary syndrome, ${ }^{3}{ }^{4}$ but little is known about the regulation of EPC mobilisation in patients with stable coronary artery disease (CAD). In the present study, we investigated the number of circulating $\mathrm{CD}_{3} 4^{+}$cells in patients with $\mathrm{CAD}$ and the influence of atherosclerotic risk factors on this number.

\section{METHODS}

Thirty four patients (mean (SEM) age 62.5 (1.7) years) with angiographically documented stable CAD, and 36 healthy control subjects (mean (SEM) age 53.6 (2.0) years) without any evidence of CAD by history and physical examination, were enrolled in the present study. Risk factors for CAD were defined as: a history of hypertension for more than one year that required the initiation of antihypertensive treatment; a history of smoking more than one pack per year and currently smoking; hyperlipidaemia, defined as total cholesterol concentrations exceeding $5.70 \mathrm{mmol} / \mathrm{l}$; and diabetes

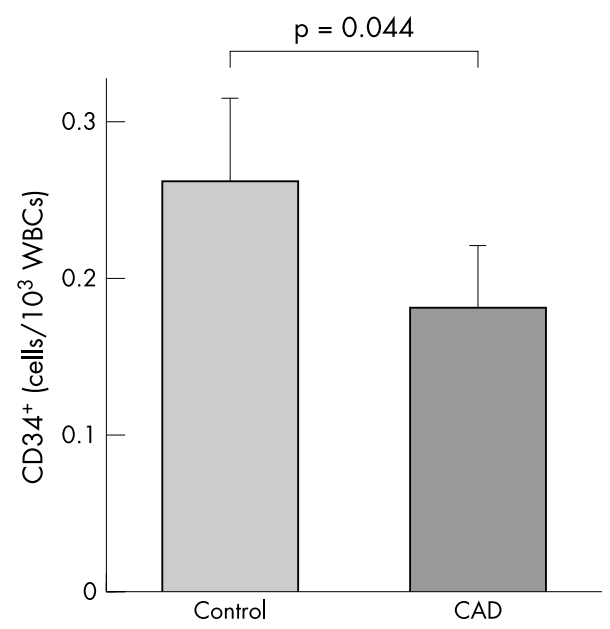

Figure 1 The number of circulating $\mathrm{CD} 34^{+}$cells. $\mathrm{CD} 34^{+}$cells were detected in peripheral blood from patients with $C A D(n=34)$ and healthy control subjects $(n=36)$. mellitus, defined as the need for oral anti-diabetic drug treatment or insulin use. The study protocol was approved by the ethics committee of Jichi Medical School, and informed consent was obtained from all patients and control subjects.

The number of $\mathrm{CD} 34^{+}$cells in white blood cells (WBCs) was quantified by FACScan (Becton-Dickinson). In brief, WBCs were stained with a fluorescein isothiocyanate conjugated anti-CD34 monoclonal antibody (BectonDickinson, Franklin Lakes, New Jersey, USA). The samples were subjected to a two dimensional side scatter fluorescence dot plot analysis. After appropriate gating, the number of CD $34^{+}$cells with low cytoplasmic granularity (low sideward scatter) was quantified and expressed as the number of cells per $10^{3}$ WBCs.

Values were expressed as mean (SEM). Significance was evaluated using unpaired Student's $t$ test for comparisons between two means. The interaction between the number of $\mathrm{CD}_{3} 4^{+}$cells and risk factors was examined by multivariate analysis using the multiple stepwise logistic regression model. Differences of $\mathrm{p}<0.05$ were considered significant.

\section{RESULTS}

The number of $\mathrm{CD} 34^{+}$cells in the peripheral blood of patients with CAD and control subjects was determined by flow cytometry. As shown in fig 1 , circulating CD $34^{+}$cells were significantly reduced, by approximately $30 \%$, in patients with CAD compared with age matched control subjects.

We then investigated the influence of risk factors on the number of circulating $\mathrm{CD}_{3} 4^{+}$cells. As shown in fig 2, univariate analysis identified diabetes mellitus as a significant predictor of a reduced $\mathrm{CD} 34^{+}$cell count. In contrast, the number of $\mathrm{CD}_{3} 4^{+}$cells did not significantly differ when patients were stratified according to sex, hypertension, hyperlipidaemia, and smoking.

Multivariate analysis also demonstrated that diabetes mellitus is a significant independent predictor of a reduced circulating $\mathrm{CD}^{2} 4^{+}$cell count (standard coefficient -0.285 , $\mathrm{p}=0.027$ ), whereas other factors were not significant.

\section{DISCUSSION}

The results of the present study demonstrate that the number of EPCs, as measured by the number of cells expressing CD34, was significantly reduced in patients with CAD compared with control subjects. Analysis of the individual risk factors indicated that subjects with diabetes mellitus had reduced numbers of circulating $\mathrm{CD}_{3} 4^{+}$cells. Given that several experimental studies indicate a significant contribution of EPCs for adult neovascularisation, the reduction in the number of $\mathrm{CD}_{3} 4^{+}$cells might contribute to reduced vascularisation in patients with CAD.

Abbreviations: $C A D$, coronary artery disease; EPC, endothelial progenitor cell; WBC, white blood cell 

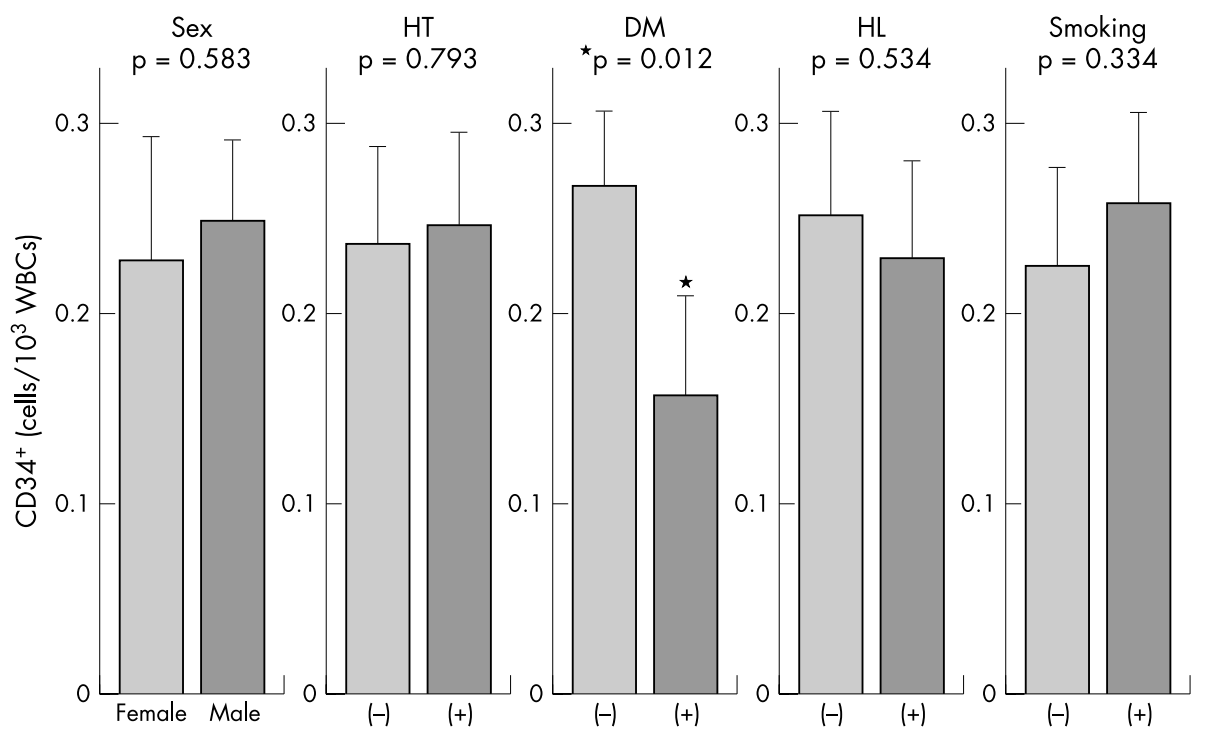

Figure 2 Effect of individual risk factors on the number of CD34 cells. DM, diabetes mellitus; $\mathrm{HL}$, hyperlipidaemia; HT, hypertension.

The mechanisms by which diabetes mellitus reduces $\mathrm{CD} 34^{+}$ cell numbers remain to be determined. There are several possible scenarios by which diabetes mellitus could reduce the number of circulating $\mathrm{CD} 34^{+}$cells. One explanation might be increased apoptosis of premature progenitor cells. Indeed, CD $34^{+}$cells were shown to be very sensitive to apoptosis induction $^{5}$ and diabetes mellitus is known to increase oxidative stress, a well established stimulus for apoptotic cell death. Alternatively, diabetes mellitus may interfere with the signalling pathways that regulate EPC differentiation or mobilisation. The present results might be related to premature atherosclerosis and impaired collateralisation in diabetes mellitus, although further studies are required to prove this hypothesis.

In conclusion, the present study demonstrates that $\mathrm{CD} 34^{+}$ cell numbers are impaired in patients with $\mathrm{CAD}$ and this impairment is related to diabetes mellitus.

\section{Authors' affiliations}

T Eizawa, Y Murakami, K Matsui, K Shimada, Divisions of Cardiovascular Medicine, Jichi Medical School, Tochigi, Japan
U lkeda, T Yoshioka, M Takahashi, Department of Organ Regeneration, Shinshu University Graduate School of Medicine, Matsumoto, Japan K Muroi, Cell Transplantation and Transfusion, Jichi Medical School

Correspondence to: Dr Uichi lkeda, Department of Organ

Regeneration, Shinshu University Graduate School of Medicine, 3-1-1 Asahi, Matsumoto 390-8621, Japan; vikeda@sch.md.shinshu-u.ac.jp

Accepted 6 November 2002

\section{REFERENCES}

1 Asahara T, Murohara T, Sullivan A, et al. Isolation of putative progenitor endothelial cells for angiogenesis. Science 1997;275:964-7.

2 Tateishi-Yuyama E, Matsubara H, Murohara T, et al. Therapeutic angiogenesis for patients with limb ischaemia by autologous transplantation of bone-marrow cells: a pilot study and a randomised controlled trial. Lancet 2002;360:427-35.

3 Shintani S, Murohara T, Ikeda H, et al. Mobilization of endothelial progenitor cells in patients with acute myocardial infarction. Circulation 2001; 103:2776-9.

4 Vasa M. Fichtlscherer S, Aicher A, et al. Number and migratory activity of circulating endothelial progenitor cells inversely correlate with risk factors for coronary artery disease. Circ Res 2001;89:el-e7.

5 Ito H, Rovira II, Bloom ML, et al. Endothelial progenitor cells as putative targets for angiostatin. Cancer Res 1999;59:5875-7. 